\title{
Periodicity of the Rational Numbers
}

\author{
Open Mathematics Collaboration*†
}

November 12, 2019

\begin{abstract}
In this article, we prove that $\alpha$ is a rational number if, and only if, its decimal representation possesses a period.
\end{abstract}

keywords: rational numbers, periodicity, irrational numbers.

\section{Preliminaries}

1. A real number $\alpha$ is called rational if we can write $\alpha=\frac{p}{q}$ for some integers $p$ and $q \neq 0$.

2. There are infinitely many real numbers that are not rational. We call them irrational.

3. For example, $\sqrt{2}$ and $\pi$ are irrational numbers (see 11 , Theorem 6.18).

4. Consider $\alpha=a_{0} . a_{1} a_{2} a_{3} \cdots$ the decimal representation of some real number $\alpha$, where $a_{0}$ is an integer and $a_{1}, a_{2} \cdots$ belong to $\{0,1,2,3,4,5,6,7,8,9\}$.

*All authors with their affiliations appear at the end of this paper.

†Corresponding author: jc.oliveira@uft.edu.br | Join the Open Mathematics Collaboration 
5. The representation (4) possesses a period if there exist two natural numbers $n$ and $m$ such that $a_{n} a_{n+1} \cdots a_{n+m}=a_{n+m+1} a_{n+m+2} \cdots a_{n+2 m+1}=a_{n+2 m+2} a_{n+2 m+3} \cdots a_{n+3 m+2}=\cdots$.

6. Here, $\mathbb{N}=\{1,2,3, \cdots\}$ is the set of the natural numbers.

7. The numbers $0.12334433443344 \cdots$ and $6.1222 \cdots$ possess a period.

8. The number $0.112123123412345 \cdots$, in which its decimal places are given by $1,12,123,1234,12345$ and so on, does not possess a period.

9. Finite decimal representations always have a period.

\section{Proof of the Main Result}

\section{Every real number that possesses a period is rational.}

11. Indeed, let $\alpha=a_{0} \cdot a_{1} a_{2} a_{3} \cdots$ be a nonnegative real number, as in (4), that possesses a period.

12. From definition (5), there exist two natural numbers $n$ and $m$ such that

$$
a_{n} a_{n+1} \cdots a_{n+m}=a_{n+m+1} a_{n+m+2} \cdots a_{n+2 m+1}=\cdots .
$$

13. Therefore, we write

$$
\alpha=a_{0}+0 . a_{1} a_{2} a_{3} \cdots a_{n-1}+0.0 \cdots 0 a_{n} a_{n+1} \cdots a_{n+m} \cdots .
$$

14. Applying hypothesis (11) in (13), we get

$$
\alpha=a_{0}+0 . a_{1} a_{2} \cdots a_{n-1}+a_{n} a_{n+1} \cdots a_{n+m}\left(\frac{1}{10^{n+m}}+\frac{1}{10^{n+2 m+1}}+\cdots\right) .
$$


15. It holds $\left(\frac{1}{10^{n+m}}+\frac{1}{10^{n+2 m+1}}+\cdots\right)=\frac{1}{10^{n+m}}\left(1+\frac{1}{10^{m+1}}+\frac{1}{10^{2 m+2}}+\cdots\right)$

$$
=\frac{1}{10^{n+m}}\left[1+\frac{1}{10^{m+1}}+\frac{1}{\left(10^{m+1}\right)^{2}}+\frac{1}{\left(10^{m+1}\right)^{3}}+\cdots\right] \in \mathbb{Q} \text {. }
$$

16. Clearly, (15) proves (10).

17. Conversely, every rational number $\alpha=\frac{p}{q}$ possesses a period.

18. In fact, without lost of generality, we may assume $0<p<q$.

19. There exists a natural number $k_{1}$ such that $10^{k_{1}-1} p<q \leq 10^{k_{1}} p$.

20. To prove (19), let $X=\left\{k \in \mathbb{N} ; 10^{k-1} p<q\right\} \subset \mathbb{N}$.

21. So, since $p<q$, one has $1 \in X$. Therefore, $X \neq \varnothing$.

22. We also have that $X$ is finite.

23. By the Principle of Mathematical Induction (see Exercise 2.4 in Chapter 1 from [2]), one can show that there exists $k_{1} \in X$ satisfying (19).

24. Write $\frac{p}{q}=\frac{1}{10^{k_{1}}} \frac{10^{k_{1}} p}{q}$.

25. By Euclidean Algorithm (see [3]), there are integers $r_{1}$ and $q_{1}$, with $0 \leq r_{1}<q$, such that $10^{k_{1}} p=q_{1} q+r_{1}$.

26. By 24$), \frac{p}{q}=\frac{1}{10^{k_{1}}}\left(q_{1}+\frac{r_{1}}{q}\right)$.

27. If $r_{1}=0$, there is nothing to do and (17) is easily true.

28. Otherwise, there is another natural number $k_{2}$ such that $10^{k_{2}-1} r_{1}<q \leq$ $10^{k_{2}} r_{1}$. 
29. We have $\frac{p}{q}=\frac{1}{10^{k_{1}}}\left(q_{1}+\frac{r_{1}}{q}\right)=\frac{1}{10^{k_{1}}}\left(q_{1}+\frac{10^{k_{2}} r_{1}}{q} \frac{1}{10^{k_{2}}}\right)$.

30. One more time, by Euclidean Algorithm, there are integers $r_{2}$ and $q_{2}$, with $0 \leq r_{2}<q$, such that $10^{k_{2}} r_{1}=q_{2} q+r_{2}$.

31. By 29, $\frac{p}{q}=\frac{1}{10^{k_{1}}}\left(q_{1}+\frac{1}{10^{k_{2}}}\left(q_{2}+\frac{r_{2}}{q}\right)\right)$.

32. If $r_{2}=0$, then (17) is easily true.

33. Otherwise, we repeat the arguments as from (28) to ensure the existence of a sequence of integer numbers $k_{n}, q_{n}$ and $r_{n}$ such that

$$
\frac{p}{q}=\frac{1}{10^{k_{1}}}\left[q_{1}+\frac{1}{10^{k_{2}}}\left(q_{2}+\frac{1}{10^{k_{3}}}\left[q_{3}+\cdots+q_{n-1}+\frac{1}{10^{k_{n}}}\left(q_{n}+\frac{r_{n}}{q}\right)\right]\right)\right] .
$$

34. If we repeat $n=q+1$ times the arguments done in (33), since $0 \leq r_{n}<q$ for all $n$, there exist at least two different natural numbers $i<j$, such that $r_{i}=r_{j}$.

35. So, the process from $r_{j}$ will repeat all the correspondent values of $k_{s}, q_{s}$ and $r_{s}$ that previously appear since $r_{i}=r_{j}$.

36. 35 shows exactly the period of $\alpha=\frac{p}{q}$ and proves 17 .

\section{Final Remarks}

37. The number $0.1234567891011121314 \cdots$ is not rational because it does not have a period.

38. $\pi=3.141592653589 \cdots$ does not have a period because it is not a rational number.

39. A didactic illustration of the proof of a particular case of the claim (17) is the following. 


$$
\begin{aligned}
& \underset{r_{1} 0 \ldots 0}{p} \frac{\boldsymbol{q}}{0, q_{1} 0 \ldots 0 q_{2} 0 \ldots 0 \ldots q_{n} 0 \ldots 0 q_{1} 0 \ldots 0 q_{2} \ldots} \\
& r_{2} 0 \ldots 0 \\
& \vdots \\
& r_{n} 0 \ldots 0 \\
& r_{1} 0 \ldots 0 \\
& r_{2} 0 \ldots 0 \\
& \vdots
\end{aligned}
$$

\section{Open Invitation}

Please review this article, add content, and join the Open Mathematics Collaboration. Contact jc.oliveira@uft.edu.br.

\section{Ethical conduct of research}

This original work was pre-registered under the OSF Preprints [4], please cite it accordingly [5]. This will ensure that researches are conducted with integrity and intellectual honesty at all times and by all means.

\section{References}

[1] Niven, I., Zuckerman, H. S. and Montgomery, H. L. R. An Introduction to the Theory of Numbers. 5th ed., New York, 1991.

[2] Lima, E. L. Análise Real. Volume 1, Rio de Janeiro, IMPA, Coleção Universitária, 1989.

[3] Domingues, Hygino H. and Iezzi, Gelson. Álgebra Moderna. 4th ed., São Paulo, Atual, 2003.

[4] COS. Open Science Framework. https://osf.io 
[5] de Oliveira Junior, José C. "Periodicity of the Rational Numbers". OSF Preprints, 20 Aug. 2019. https://doi.org/10.31219/osf.io/gwzhv.

\section{The Open Mathematics Collaboration}

José Carlos de Oliveira Junior (lead author, jc.oliveira@uft.edu.br)

Matheus Pereira Lobo (mplobo@uft.edu.br)

Federal University of Tocantins (Brazil) 